\title{
Natural antisense RNAs as mRNA regulatory elements in bacteria: a review on function and applications
}

\author{
Fatemeh Saberi ${ }^{1}$, Mehdi Kamali ${ }^{2}$, Ali Najafi ${ }^{1}$, Alavieh Yazdanparast ${ }^{3}$ and Mehrdad Moosazadeh Moghaddam ${ }^{3 *}$
}

\author{
* Correspondence: mm.genetics@ \\ gmail.com; abrch@bmsu.ac.ir \\ ${ }^{3}$ Applied Biotechnology Research \\ Center, Baqiyatallah University of \\ Medical Sciences, Tehran, Iran \\ Full list of author information is \\ available at the end of the article
}

\begin{abstract}
Naturally occurring antisense RNAs are small, diffusible, untranslated transcripts that pair to target RNAs at specific regions of complementarity to control their biological function by regulating gene expression at the post-transcriptional level. This review focuses on known cases of antisense RNA control in prokaryotes and provides an overview of some natural RNA-based mechanisms that bacteria use to modulate gene expression, such as mRNA sensors, riboswitches and antisense RNAs. We also highlight recent advances in RNA-based technology. The review shows that studies on both natural and synthetic systems are reciprocally beneficial.
\end{abstract}

Keywords: Bacteria, Natural antisense, Cis-asRNA, Trans-asRNA, Regulation, Riboswitch, Hfa protein, RNA engineering, RNA silencing, RNA thermosensors

\section{Background}

\section{Regulatory antisense RNA}

Antisense RNAs (asRNAs), also referred to as natural regulatory RNAs, are small molecules that perform their regulatory function by recognizing sequence and structural elements that are present in themselves and their target mRNAs. AsRNA-mediated regulation generally inhibits mRNA transcription and/or translation or induces their rapid degradation. In fewer cases, asRNAs activate expression of mRNAs [1, 2].

We are using the terms regulatory asRNAs and natural regulatory RNAs to mean a diverse group of bacterial RNAs that modulate a broad range of physiological responses through complicated and delicate controlling mechanisms [3]. Although natural regulatory RNAs were first discovered in bacteria in 1967, their importance and prevalence have not been appreciated for till recently [4]. These natural regulatory RNA sequences or transcripts are similar to the eukaryotic microRNAs (miRNAs) in terms of function and properties. However, bacteria do not possess RNA interference machinery (RNAi) per se. Bacterial asRNAs and eukaryotic miRNAs both target mRNAs to regulate their translation and/or degradation $[1,5]$.

In recent years, the prevalence of long non-coding RNAs (lncRNA) and natural antisense transcripts (NATs) has been reported in a variety of organisms. In general, antisense transcript regulatory mechanisms affect different levels of gene expression including: transcription interference, transcription attenuation, translation stimulation or inhibition,

(C) 2016 The Author(s). Open Access This article is distributed under the terms of the Creative Commons Attribution 4.0 International License (http://creativecommons.org/licenses/by/4.0/), which permits unrestricted use, distribution, and reproduction in any medium, provided you give appropriate credit to the original author(s) and the source, provide a link to the Creative Commons license, and indicate if changes were made. The Creative Commons Public Domain Dedication waiver (http://creativecommons.org/ publicdomain/zero/1.0/) applies to the data made available in this article, unless otherwise stated. 
and RNA stability [6]. Another major discovery found in bacteria was that many regulatory RNAs dwell in mRNAs [1].

Here are some of the characteristics of this type of RNA.

- Antisense RNA can be inherited during conjugation (horizontal gene transfer).

- High-throughput transcriptomics has shown that every organism can potentially possess one or several asRNA regulators for every single gene.

- New asRNAs evolve easily and automatically during mutations of the original DNA template.

- Because both antisense and target RNAs are transcribed in close proximity due to the position of the cis on their templates, a high local concentration of both types of molecule takes place. These so-called steric effects and the limited diffusion of transcripts lead to an efficient interaction between antisense and target RNAs, which is suggested to be controlled and biologically effective.

- The spatial closeness of the promoter sites to antisense and target RNAs also causes transcriptional interference, and thus has a regulatory role.

On the other hand, asRNAs can also be defined as endogenous RNA molecules containing complementary sequences to the original transcripts (mRNAs). These endogenous asRNAs have been observed widely in both prokaryotes and eukaryotes [7].

\section{cis- and trans-antisense RNAs}

Studies have shown that some asRNAs regulate protein function, mimicking the nucleic acids that are the regulatory targets of the protein, so they involve the protein in an inactive complex, whereas the majority of asRNAs pair with target mRNAs and shift their stability and/or translation [8]. In bacteria, these base-pairing/non-coding regulatory RNAs are generally subdivided into three groups: (i) cis-acting 5' element non-coding asRNAs; (ii) trans-acting small non-coding asRNAs; and (iii) cis-encoded asRNAs.

A cis-acting 5' non-coding asRNA is usually attached to the 5' side of an mRNA, the expression of which is regulated by the non-coding RNA. A structural change in the noncoding RNA occurs through binding to small metabolites (riboswitches) or through change of temperature (thermoregulators) or $\mathrm{pH}$ ( $\mathrm{pH}$ sensors). The structural change influences the transcription or translation of the downstream gene or genes in an operon.

Trans-acting small non-coding asRNAs are usually encoded in intergenic regions on the chromosome and control translation or degradation of their target mRNAs. Generally, each trans-acting non-coding asRNA has multiple target mRNAs and binds near the ribosomal binding site of the target mRNAs.

Cis-acting asRNA is expressed as a complementary sequence of mRNA that becomes the sole target RNA. Therefore, cis- and trans-acting non-coding asRNAs are a major part of the asRNAs in bacteria (Fig. 1).

Prokaryotes also have cis-encoded asRNAs. They significantly contribute to different biological procedures, such as genomic imprinting, circadian rhythm, cardiac gene regulation and recombination of antigen receptor genes [7, 9].

Some principal features of cis- and trans-asRNAs are summarized in Table 1. As implied therein, cis-asRNAs act on their targets through complete or near-complete complementarity, while short regions of complementarity suffice for trans-asRNAs to affect 


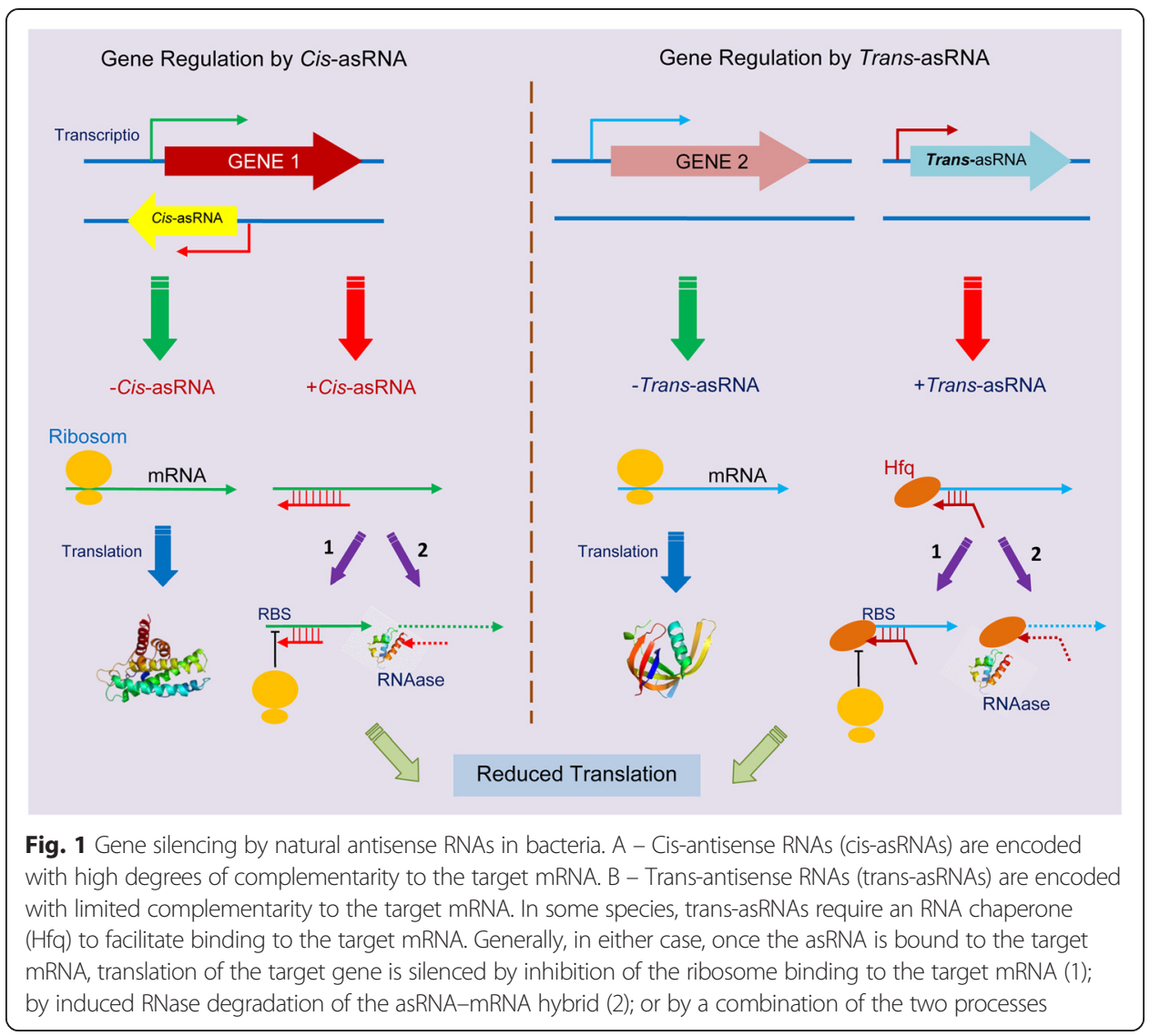

their target mRNAs. Therefore, cis-asRNAs form much more energetically stable duplexes with their targets than trans-asRNAs. Generally, cis-asRNAs are associated with mobile genetic elements such as plasmids, phages and transposons. They contribute significantly to the regulation of plasmid copy number, conjugation, phage life cycle and transposition. Several anti-toxicity activities have also been reported for some cis-asRNAs $[1,10]$.

Cis-asRNAs were first detected in viruses, then in prokaryotes and finally in eukaryotes. According to their relative orientation and degree of overlap, they are classified into 3 categories; head-to-head ( $5^{\prime}$ to $\left.5^{\prime}\right)$, tail-to-tail ( $3^{\prime}$ to $3^{\prime}$ ) and fully overlapping. Tail-to-tail orientation seems to be the most common type. Overlapping transcripts might comprise two protein-encoding genes, one protein-encoding and one nonencoding gene, or two non-encoding transcripts [11].

Table 1 Differentiation of cis- and trans-asRNAs

\begin{tabular}{ll}
\hline cis-asRNAs & trans-asRNAs \\
\hline Also known as cis-NATs or cis effector asRNAs & Also known as trans-NATs or trans effector asRNAs \\
Transcribed from the opposing DNA strand of the & Transcribed from a separate locus to the target gene \\
target gene at the same genomic locus & \\
Display perfect or extensive sequence & Display imperfect or short stretches of \\
complementarity with the target gene & complementarity with the target gene \\
Target individual mRNAs & $\begin{array}{l}\text { Target multiple sense targets and form complex } \\
\text { regulation networks }\end{array}$ \\
Mostly short & $\begin{array}{l}\text { Frequently longer: several hundred nt, in some } \\
\text { examples several kb long }\end{array}$ \\
\hline
\end{tabular}


Based on recent studies, one of the popular cis-acting regulatory regions of the mRNAs acts as a potential target for the development of novel anti-bacterial compounds or as a regulatory factor for biotechnological applications due to versatile properties of the RNAs and their ability to be regulated by physical parameters (thermosensors) or small molecules (riboswitches). Other high-throughput screens have made it possible to design artificial metabolite analogs that modulate trans-acting regulatory RNAs with two functions: for example, a riboswitch coupled to an asRNA [12].

Thus, it is known that asRNAs regulate prokaryotic and eukaryotic gene expression at multiple levels including transcription, RNA editing, post-transcription and translation. They show such a wide distribution and conservation that the assumption of their accidental existence can be completely disclaimed (Table 2) [13-16].

Trans-asRNAs are generally much longer than the cis-asRNAs. It follows that the promoter and first transcribed nt of asRNAs may play the part of an intergenic spacer and then extend to a complementary region. However, cis-asRNAs form more stable complementary duplexes because of their binding kinetics. In addition, cis-asRNAs seem to exert their target interaction independently of proteins such as Hfq (Table 2), but other protein factors such as RNA helicases and RNA binding proteins are involved in the association between sense and antisense RNA [13].

The sizes of asRNAs are also very diverse. There are examples of rather short NATs of only 100 to 300 nt e.g., SymR, GadY and SyR7, but many asRNAs are substantially longer, ranging from 700 to 3,500 nt. At least one example, in Prochlorococcus sp. strain

Table 2 Some mechanisms used by bacterial antisense RNAs [16]

\begin{tabular}{|c|c|c|c|c|}
\hline Antisense/target & Organism & Mechanism & Description & $\begin{array}{l}\text { Needs } \\
\mathrm{Hfq}\end{array}$ \\
\hline MicA/ompA & Escherichia coli & $\begin{array}{l}\text { Direct }(T I R) \text { inhibition } \\
\text { of translation }\end{array}$ & $\begin{array}{l}\text { Trans-encoded; entails RNase } \\
\text { E-dependent mRNA decay }\end{array}$ & Yes \\
\hline SgrS/ptsG & E. coli & $\begin{array}{l}\text { Direct }(T I R) \text { inhibition } \\
\text { of translation }\end{array}$ & $\begin{array}{l}\text { Trans-encoded; SgrS also encoded } \\
\text { o protein }\end{array}$ & Yes \\
\hline CopA/CopT & $\begin{array}{l}\text { E. coli R1-type } \\
\text { plasmids }\end{array}$ & Inhibition of translation & $\begin{array}{l}\text { Cis-encoded; involves translational } \\
\text { coupling }\end{array}$ & No \\
\hline IstR1/tisB & E. coli & Inhibition of translation & $\begin{array}{l}\text { Trans-encoded; binding upstream } \\
\text { of TIR inhibits standby ribosomes }\end{array}$ & No \\
\hline RNAIII/sa1000 & $\begin{array}{l}\text { Staphylococcus } \\
\text { aureus }\end{array}$ & $\begin{array}{l}\text { Inhibition of translational } \\
\text { initiation and induced } \\
\text { RNase III cleavage }\end{array}$ & $\begin{array}{l}\text { Trans-encoded; full inhibition requires } \\
\text { both translation block and endocleavage; } \\
\text { also contain open reading frame }\end{array}$ & No \\
\hline DsrA/rpos & E. coli & $\begin{array}{l}\text { Activation of Rpos } \\
\text { translation }\end{array}$ & $\begin{array}{l}\text { Trans-encoded; binding upstream } \\
\text { of TIR }\end{array}$ & Yes \\
\hline RNAIII/repR & $\begin{array}{l}\text { Streptococci } \\
\text { plasmid plP501 }\end{array}$ & $\begin{array}{l}\text { Riboswitch-like induction } \\
\text { of premature transcription } \\
\text { termination }\end{array}$ & Cis-encoded & $?$ \\
\hline GadY/gadXY & E. coli & $\begin{array}{l}\text { Induced cleavage, } \\
\text { stabilization of gadX mRNA }\end{array}$ & $\begin{array}{l}\text { Cis-encoded between gadX } \\
\text { and gadY }\end{array}$ & Yes \\
\hline $\mathrm{OOP} / \mathrm{cll}$ & $\begin{array}{l}\text { E. coli Lambda } \\
\text { phage }\end{array}$ & $\begin{array}{l}\text { Induced RNase III-mediated } \\
\text { cleavage near } 3^{\prime} \text { end }\end{array}$ & $\begin{array}{l}\text { Cis-encoded; causes subsequent } \\
\text { cll mRNA decay }\end{array}$ & $?$ \\
\hline MicC/ompD & Salmonella & $\begin{array}{l}\text { Direct targeting for mRNA } \\
\text { decay }\end{array}$ & $\begin{array}{l}\text { CDS-internal target; no effect on } \\
\text { translational initiation }\end{array}$ & Yes \\
\hline RNAI/RNAll & $\begin{array}{l}\text { E. coli ColE1- } \\
\text { type plasmids }\end{array}$ & $\begin{array}{l}\text { Inhibition of replication } \\
\text { primer maturation }\end{array}$ & $\begin{array}{l}\text { Cis-encoded; induces RNA folding } \\
\text { change }\end{array}$ & No \\
\hline $\begin{array}{l}\text { crRNAs/phage } \\
\text { RNA/DNA? }\end{array}$ & $\begin{array}{l}\text { Bacteria and } \\
\text { archea }\end{array}$ & $\begin{array}{l}\text { Mechanism is homology- } \\
\text { dependent but unknown }\end{array}$ & $\begin{array}{l}\text { CrRNAs processed from precursor } \\
\text { RNA; anti-phage immunity }\end{array}$ & $?$ \\
\hline
\end{tabular}


MED4, is 3500 and/or 7,000 nt, overlapping 14 genes of a ribosomal protein operon. These asRNAs protect a set of mRNAs that accumulate during phage infection from RNase E degradation. It is noteworthy that this type of asRNA-mRNA duplex formation masks single-stranded recognition sites of RNase E, leading to increased stability of the mRNAs during phage infection $[17,18]$.

In this review, we will focus our discussion on bacterial small RNAs that act as regulators. In general, the aim of this review is to provide an overview of some natural RNA-based mechanisms, such as mRNA sensors, riboswitches and asRNAs, which are used by bacteria to modulate gene expression. We would also like to highlight recent advances in RNA-based technology. This review emphasizes how studies on natural and synthetic systems are reciprocally beneficial.

\section{Antisense RNAs in bacteria - discovery and history}

The first natural E. coli asRNA (micF RNA) was identified during the characterization of the $E$. coli outer membrane porin ompC. During that study, it was noticed that several ompC promoter clones repressed the synthesis of the other major outer membrane porin OmpP. The responsible region for this activity turned out to be a 300-bp fragment located upstream of the ompC promoter, $70 \%$ homologous to the DNA encoding the 5' end of the ompF mRNA (including the ribosome binding site and the ompF initiation codon). Further studies showed that this homologous 300-bp fragment is transcribed in the opposite direction from ompC to a 174-nucleotide RNA that complements and encompasses a region of the ompF mRNA containing its translation initiation site. Based on this study, it was proposed that micRNA forms a hybrid with ompF mRNA and inhibits its expression by preparing a model that seeded the idea of using artificial micRNAs to regulate selected genes $[19,20]$.

Studies have shown that the micF gene produced this asRNA. micF is a stress response gene found in $E$. coli and related bacteria. It post-transcriptionally controls the expression of the outer membrane porin gene ompF by encoding a trans-asRNA (93 nt) that binds its target ompF mRNA and regulates ompF expression by inhibiting translation and inducing degradation of the message [21].

Several mapping, cloning, sequencing and bioinformatics techniques and tools have helped to identify and characterize asRNAs, but various aspects of their functions, structures and mechanisms of action remain to be explored. Accordingly, fundamental and mechanistic studies aiming to characterize novel regulatory RNA are needed if biotechnological and medical aims are to be met [22].

Although regulatory RNAs and their mechanisms were discovered in prokaryotes in 1967 , it is only now that the central role of RNA in prokaryote gene regulation in all three domains of life can be demonstrated. The research that led to this discovery occurred in three major phases: 1967 to early 2001; 2001 to recent times; and current research [13, 23].

In 1967, Hindley identified a distinct and abundant RNA species, later named $6 \mathrm{~S}$ RNAI. Its function in RNA polymerase activity regulation had been determined previously. The first trans-acting regulatory RNAs were discovered around this time [4].

Initially, it was thought that cis-asRNAs controlled the life cycle or copy number of extra-chromosomal genetic elements, bacteriophages, transposons and plasmids. One of the first identified antisense transcripts belonged to the gene cro in bacteriophage $\lambda$. 
It was revealed to be transcribed by bacteriophage $\lambda$ when overexpression of the $77 \mathrm{nt}$ OOP antisense transcript resulted in its co-degradation with the cII mRNA [24]. Another extra-chromosomal element was a cis-asRNA I involved in the regulation of maturation of the ColE1 primer and the control of plasmid incompatibility of ColE1-type plasmids.

Over the next two decades, although a small number of chromosomally encoded small RNA regulators such as MicF, DicF and OxyS were discovered, their fundamental importance and privileges were not adequately considered. Only 12 small RNAs containing entities like 6S RNA, tmRNA, RNase P RNA and 4.5 S RNA had been identified in E. coli by early 2001.

In 2001, considerable progress took place thanks to the application computational techniques and predictive approaches to complex systematic experimental screens. For example, comparative genome analysis of closely related species was used for searching transcriptional signals within intergenic regions and scoring the conservation of predicted RNA secondary structure with the aim of small RNA prediction in Enterobacteria. During this second phase, newly identified trans-acting asRNAs supplied abundant data for later functional characterization studies [25].

The advent of RNA-seq technology and its use in prokaryotic RNA studies triggered a new wave of discovery. This has led to new standards in the accelerated identification of transcripts and transcriptional start sites $[13,26]$.

\section{Why antisense regulation?}

Ongoing studies present two reasons for the advantage of asRNA regulation over other methods of regulation. First, asRNAs could provide an advantageous control system when a particular protein such as transposase needs to be expressed under very selective circumstances or repressed at a very tight level. Second, asRNAs provide one more level of control in the extensive regulation of their targets. For example, translation and expression of $\mathrm{MgtC}$, a virulence factor of Salmonella enterica, is regulated at the transcriptional level by PhoPQ, and at the level of protein stability by the MgtR peptide. Additionally, it might be regulated at the post-transcriptional level by the AmgR asRNA [27].

In this way, the advantages of RNA-based regulation over classical protein-mediated transcriptional control can be referred as:

1. The response to the regulator happens in a shorter time because in this way mRNA is a direct target.

2. In the case of small RNAs, a low number of base pairs provide specific and fast mRNA recognition and binding.

3. Rapid specificity can be attained because a single nucleotide change suffices for a specificity shift.

4. The capacity of RNA regulators to modulate their conformation upon binding increases the number of contacts with a given ligand or makes the recognition of multiple targets easier [26].

These features show the potential of artificial mRNA regulators that can be beneficial for various biotechnological applications, supporting the need for more studies [24]. 


\section{Antisense RNA functions in bacteria}

As mentioned, asRNAs affect cellular functions through transcription attenuation, translation inhibition, and regulation of plasmid copy number through the inhibition of primer maturation, prevention of formation of an activator RNA, and promotion or inhibition of mRNA degradation [16, 28]. They perform their regulatory actions by annealing to complementary mRNA strands. For example, they can block translation through steric hindrance or cause rapid degradation by dsRNA-specific RNases. The length and structure, intracellular concentration of the antisense sRNA and resistance to the degradation of the target mRNA influences the proficiency of degradation $[13,29]$. A few examples of different asRNA mechanisms of action in prokaryotes are given below.

\section{Induction of a bacterial gene}

MucD gene expression and consequent alginate (biofilm) biosynthesis in Pseudomonas aeruginosa is regulated by cis-asRNA (named mucD_AS). Functionally, mucD_AS turned out to be able to induce biofilm formation in $P$. aeruginosa. Considering the biotechnological and medical significance of alginate as a key virulence factor of $P$. aeruginosa, this finding is particularly important [30].

\section{Inhibition of bacterial gene expression}

An asRNA named micF inhibits the expression of the membrane porin gene, ompF. This occurs under the regulation of environmental and internal stress factors, including elevated temperature, exposure to salicylate and redox stress. ompF is at its maximal expression level at low temperature and osmolarity and this is regulated at the transcription and post-transcription levels by micF asRNA [16]. However, the regulatory mechanism is not clear: it seems that micF destabilizes the ompF mRNA and inhibits its translation. It is likely that specific binding proteins are also associated to micF asRNA, while both seem conserved in other Gram-negative species. Other controlling systems are involved at different levels [31, 32].

\section{Post-transcriptional inhibition of mRNA}

dicF is an asRNA that inhibits cell division in E. coli. ftsZ mRNA, which is apparently involved in initiating division, is inhibited by several factors, including the 53-nt dicFRNA, which is transcribed from $\operatorname{dic} B$ operon (another inhibition factor) and partially complementary to the ftsZ mRNA [31].

\section{Inhibition of translation}

The expression of glutamine synthetase gene $(g \operatorname{In} A)$ is likely regulated by asRNA in Clostridium. This is an important enzyme for nitrogen assimilation in Gram-positive Clostridium acetobutylicum and is nitrogen regulated. The regulatory $43 \mathrm{nt}$ asRNA is complementary to the ginA RBS and its transcription induced in nitrogen level of environment is high leading to repressed $\operatorname{gin} A$ transcripts [31, 33].

\section{Regulating plasmid replication}

Controlling genes and sites in the replication frequency of plasmids are located on the plasmids themselves, which keeps the copy numbers of plasmids stable. Many plasmids use asRNAs to measure their own copy numbers and adjust deviations. Controlling 
other protein synthesis (such as a rate-limiting replication protein) or primer inhibition (in the case of the ColE1-type plasmids) can take place too [19, 31, 34]. Some examples are given in Table 3.

\section{Inhibition of F-like plasmid conjugation}

More than 30 tra genes are involved in the conjugation of F-like plasmids (such as Incf and R1). One of these is a transcriptional activator, traJ. It is regulated by an asRNA system, FinP, which complements to traJ RBS and blocks its translation. FinP metabolic stability increases with the help of a ribonucleolytic degradation inhibitor protein, FinO. Structurally, FinP has two stem-loops, so loops are responsible for specificity and stems and play a pivotal role in its activity $[4,14]$.

\section{Inhibition of plasmid and host-encoded killer systems}

Host-killing systems are employed by many bacterial plasmids to kill cells that fail to keep at least one plasmid copy at cell division to guarantee their maintenance in bacterial cells $[19,31]$. All members of the growing list of plasmid-borne killer systems have a similar genetic structure. They produce a small protein that increases the permeability of the cell membrane, leading to cell death. There is an asRNA that inhibits the expression of this protein at the post-translational level. An overlapping reading frame is involved in the killer mechanism and in its inhibition [35].

\section{Control of bacteriophage development}

In the bacteriophages P22 and A, antisense control exists as a secondary control strategy and contributes at various levels to their developmental pathways. Some of these RNA transcripts and their functions are listed below [31].

- OOP RNA facilitates cell mRNA decay.

- PaQ RNA inhibits late gene expression.

- P22 sar RNA inhibits anti-repression.

- Sas RNA in phage P22 regulates alternative translation.

\section{Controlling toxin synthesis}

SymE protein shows homology to MazE, an antitoxin, encoded from an SOS-induced gene. It is suggested to be involved in cell growth inhibition, decreased protein synthesis and increased RNA degradation, and therefore functionally resembles RNA endonuclease

Table 3 Plasmids using NAT regulating systems

\begin{tabular}{|c|c|}
\hline Plasmid & The role of the antisense RNA \\
\hline \multirow[t]{2}{*}{ ColE1 } & $\begin{array}{l}\text { An asRNA, termed RNA I, binds to the } 5^{\prime} \text { end of RNA II, triggering a conformational } \\
\text { change that initiates inhibiting replication by preventing persistent hybrid formation. }\end{array}$ \\
\hline & $\begin{array}{l}\text { Another asRNA, Rcd }{ }^{a} \text {, complementary to the Cer region of plasmid, is synthesized when } \\
\text { synaptic complexes between Cer sites are formed. It inhibits division when the plasmid } \\
\text { is in a multimeric state and at risk of being lost. }\end{array}$ \\
\hline IncFll-Like & $\begin{array}{l}\text { An asRNA blocks the translation of leader protein and causes inhibition of Rep protein } \\
\text { synthesis. }\end{array}$ \\
\hline Inclu-IncB & An asRNA prevents the formation of an activator RNA pseudo knot. \\
\hline pT181 and pIP501 & Antisense RNA induces attenuation of transcription. \\
\hline
\end{tabular}


toxins. SymR is one of several mechanisms that repress SymE gene expression. It contains an asRNA that is encoded in cis to the SymE gene [36, 37].

\section{Small RNAs involved in quorum-sensing and biofilm formation}

Recent studies have revealed that RNAs are key regulators in pathogens. Small RNAs regulate the translation and/or stability of mRNAs that encode virulence factors, or proteins with roles in adaptive responses, which are triggered by environmental cues and stresses. Generally, the transcription of many pathogenesis-related RNAs is dependent on the growth phase but in several pathogens, the secretion of virulence factors is regulated by cell-density sensing (quorum sensing), a process that involves communication through secreted signaling molecules. Several regulatory RNAs are the main effectors of quorum-sensing systems [38, 39].

For example, in Vibrio cholerae, the sensory signals converge on a response regulatory protein, LuxO. At low cell density, when the quorum-sensing autoinducer is absent, phosphorylated LuxO activates the transcription of four redundant Qrr RNAs as quorum regulatory RNAs (Qrr1-Qrr4) that regulate the mRNA of the downstream target gene hapR through the inhibition of translation and mRNA degradation. In Staphylococcus aureus, the effector of quorum sensing is encoded by the agr system, which is composed of two divergent transcription units. The first operon combines a density-sensing cassette including $\operatorname{agr} D$ and $\operatorname{agr} B$, and a two-component sensory transduction system (agrA and agrC), which is required for its autocatalytic activation as well as for the activation of transcription of RNAIII, the intracellular effector of the agr regulon.

Although it had previously been thought that small RNAs are noncoding, based on this research several such entities were recognized to be both regulatory and protein coding (regulatory RNA and mRNA). For example, RNAIII is one of the first identified trans-acting regulatory RNAs shown to encode a 26-amino acid peptide that may be involved in biofilm integrity in addition to its regulatory effect in quorum-sensing in $S$. aureus [40].

In E. coli, when phosphor-sugar intermediates can increase to toxic levels, SgrS, a 227-nt small RNA, represses ptsG, the glucose transporter component of the PTS, at the translational level. SgrS encodes for a 43-amino acid oligo-peptide, SgrT, which inhibits glucose transportation of PtsG. Transcriptional profiling has revealed five potential sRNAs in Listeria monocytogenes. They are suggested to encode 28-to 64-amino acid small peptides [41].

\section{A brief overview of other regulatory RNA elements}

Many other kinds of mRNA regulatory elements exist that are actually located on the same strand and near the locus of target mRNA, just upstream of the target coding sequence. These types of regulatory elements are also classified as cis-acting RNA regulators [42].

\section{Metabolite-sensing riboswitches}

Some regulatory mRNAs modulate gene expression through allosteric mechanisms due to stimulant metabolites that modify the mRNA structure and activity by binding to 5' UTR regulatory elements. The modified structure of the mRNA influences the expression of the following coding sequence. These metabolite-sensing mRNAs, which are called riboswitches, are widespread in bacteria. They give feedback regulation that supplies the cell 
with a reciprocal regulation system that is responsive to the concentration of stimulant metabolites. In addition, the synergism of different riboswitches in functional units develops even more complex regulatory arrangements. Such metabolite-specific tandem riboswitches may act independently or associate together $[1,12]$.

\section{RNA thermosensors}

RNA molecules have a particular potential to sense temperature due to their versatility and dynamism. Several mRNAs of heat- and cold-shock proteins also act as thermosensors. It is shown that some proteins associated with bacterial pathogenesis or bacteriophage development and lysogeny have mRNAs that are regulated by the thermosensor system. As with the riboswitches, the thermosensor regulatory elements, are settled at the 5' UTR of mRNAs. Another mutual mechanism controls all heat- and cold-shock responsive elements and translation initiation, so that low temperature causes target mRNA RBS to fold into a helical structure preventing the formation of the ribosomal initiation complex and protein synthesis initiation. However, in higher temperature, the inhibitory structure unfolds and then protein synthesis begins after ribosomal initiation complex formation.

In many Gram-negative bacteria, this 5' UTR regulatory element of heat-shock protein mRNAs contains 4 hairpins. It has the name ROSE (repression of heat-shock gene expression). Another example is the 5' UTR of prfA mRNA in Listeria monocytogenes, which contains a similar hairpin that melts at host temperature $\left(37^{\circ} \mathrm{C}\right)$, permitting the protein to start its virulence function. Some other thermosensitive elements may adopt more stable structures. Thus, thermosensitive regulatory activity is influenced by the rate of the transcription, the thermosensitive folding performance and the ribosome recognition efficiency of these structures [1].

\section{Hfa-associated regulatory small RNAs}

As implied before, thermosensors and riboswitches were from well-characterized groups of cis-acting regulatory sRNAs. Trans-acting regulatory RNAs are the best-characterized class of RNA regulators [43, 44]. Trans-encoded asRNAs are extensively observed in bacterial genomes and show partial complementarity to their target. The pleiotropic regulator protein Hfq is a highly conserved hexameric protein that tends to bind to an AU-rich region of a single-strand RNA preferentially close to a stem-loop structure. It was recently found in association with many trans-encoded regulatory RNAs, regulating the mRNA translation $[45,46]$. Most of the Hfq-dependent small RNAs contain 3 structural partitions: a 5'-targeting domain, a Hfq-binding domain and a 3'-located terminator [28].

\section{Synthetic antisense RNA}

An antisense RNA regulatory system is potentially very useful for metabolic engineering and/or as a therapeutic agent in prokaryotic organisms [27]. The understanding of regulatory asRNAs and their mechanisms enable us to design synthetic RNA regulatory systems for a variety of biotechnological and medical purposes. Artificial regulatory RNAs have aided many biological studies, such as in the identification of riboswitches in bacteria.

In some studies, the targeting region of recognized Hfq-associated regulatory asRNAs was merged to other asRNA backbones to construct custom regulatory asRNAs with a desired regulatory function. As the library of various regulatory small RNA modules 
becomes more complete, the design of a completely recombinant asRNA becomes more approachable. Mutational techniques and in silico modeling software are helpful to predict the designed asRNAs structurally and functionally $[1,47]$. What follows are some of the existing applications.

\section{Antisense RNA as a metabolic engineering tool to enhance the productivity of several bacterial hosts}

Natural prokaryotic antisense systems were the first RNA-based regulatory systems applied in bacteria. The locus of parB partition in the plasmid R1, which stabilizes the plasmid in the carrier cell through toxin/antitoxin counteraction, is also regulated under an RNA-based regulation system. Therefore, this regulatory system might be helpful when R1 is used to produce a special product [48]. In addition, there are many other biological procedures that are controlled with asRNA-based strategies. The regulation of biosynthesis of the global regulatory proteins and metabolites in Clostridium acetobutylicum and E. coli are noteworthy examples [14].

Furthermore, asRNA strategies are suggested to enhance recombinant protein production in E. coli. One approach is to silence the RNaseE using asRNAs and decrease the cellular level of RNaseE. This is supposed to decrease mRNA degradation in the cell, resulting in augmentation of the target mRNA and the related product [49].

Regulatory asRNA was also studied in Clostridium acetobutylicum. The idea was to redirect the primary metabolism of bacteria trying to reduce the levels of the butyrate-forming enzymes, because butyrate is assumed to induce solvent genesis in C. acetobutylicum. The larger goal was downregulation of the primary metabolism of C. acetobutylicum, which is a promising organism for biorefinery [50, 51].

Regulatory asRNA was also used in Lactobacillus rhamnosus, a milk-fermenting bacterium. The purpose was to change the polysaccharide size without any alteration to the total amount. This offers a conditional control of gene expression in metabolic engineering samples without gene inactivation [52].

\section{Antisense RNA as bacterial protection against bacteriophages}

Bacteriophages can be highly disruptive in microbial industries that use starter cultures or produce live mucosal vaccines or enzymes and metabolites. It has been shown that asRNA anneals to sense RNA and makes it subject to dsRNase enzymes. The idea of designing asRNAs against essential genes for phage development is of great interest [53].

\section{Artificial antisense RNAs as gene silencers}

Despite all of the controversial reports on the ability of asRNAs to silence genes, one study did show that they can silence a gene by decreasing target mRNA concentration [54]. Since lacZ is an ideal subject for gene silencing studies, it has been used as a target for synthetic asRNAs. The $\beta$-galactosidase activity assessment has shown to be successful in silencing lacZ in E. coli. The goal of the study in question was to survey the silencing efficiency and repression mechanism $[54,55]$.

\section{Synthetic RNA silencing as antimicrobials}

A variety of RNA-level regulatory systems have been developed in bacteria. The most important factor in their development is that mRNA can rapidly switch from repression 
to expression and vice versa in response to environmental stimulation $[56,57]$. The antibiotic resistance system is noteworthy because cell survival in the presence of antibiotics needs a rapid antibiotic resistance gene expression and cis-encoded antisense sequences provide this. These asRNA coding sequences are situated within a short open reading frame just upstream of the start codon region of both the chloramphenicol and erythromycin resistance genes $[58,59]$. RNA-silencing sequences manage gene regulation not only located out of the operon but also when they lie within the operon. For instance, Spot42 RNA, an antisense repressor sequence for gal is located within the galETKM operon. Additionally, RNA silencing acts as an RNA regulatory system in the protection of bacteria from viral infection. Natural RNA silencing provides bacteria to a wide range of RNA-level gene control [60].

\section{Antisense RNA as a tool to analyze essential genes in bacteria}

Antisense RNA regulation has been employed for determining essential genes of bacteria. $S$. aureus has been targeted by two research groups applying shotgun technology to make a library of $S$. aureus genome fragments cloned under inducible promoters aimed to essential gene identification. Although the suboptimal regulatory efficacy of RNA may contravene the desired lethal activity, the RNA regulators have been successful enough to recognize many essential genes of $S$. aureus, leading to infection resolution in the animal model $[1,61]$.

\section{ncRNAs/RNA aptamer-fused regulators}

Non-coding RNAs (ncRNAs) have a variety of regulatory functions in cell. Since most trans-acting ncRNAs generally cannot sense cellular signals directly, allosteric RNA fusion molecules have been engineered combining ncRNAs (as the target recognition motif) and RNA aptamers (as the ligand-sensing motif). The fused aptamer part enables the ncRNA modulatory activity in E. coli. This is applicable to both translation ncRNA regulators (e.g., IS10 ncRNA) and transcription ncRNA regulators (e.g., pT181 ncRNA). This reconfiguration develops an orthogonally acting fusion molecule capable of recognition of different ligands and affecting different subjects in a biological system [62-64].

Finally, the technique of aptamer-ncRNA fusion molecules provides researches with a very extended and efficient tool for ligand-sensing regulatory circuits. A single ncRNA can be fused to various aptamers as sensing domains and can bring up ligand sensor control systems that respond to multiple input signals applicable for structurally related families of ncRNAs [65].

\section{Synthetic small RNAs as ligand-specific purification tools}

For experimental investigations of small RNAs and their interactions with proteins, they must be purified in a native form. Aptamer-tagged variants of target small RNAs can be produced and used in RNA-based affinity chromatography. Thus, appropriate plasmids have been developed to express target small RNAs tagged with one of three global aptamer sequences: MS2, boxB or eIF4A [65].

\section{Synthetic RNA thermosensors}

By exploiting biomimicry, in vivo selection and computational design, synthetic thermosensors have been created. These trials made a simple on/off switch to express any 
desired gene in response to temperature. A similar principle was implemented to fuse the PrfA leader sequence to $g f p$ mRNA. It could confer temperature dependence to gene expression in E. coli [66].

Other physical stimuli can also potentially be detected by this sensor-mediated regulatory system, making it possible to apply more criteria to biological systems and offering more metabolic engineering tools to researchers [1].

RNA thermometers (RNATs) mostly regulate translation using a zipper like function and blocking the SD sequence at low temperature, resulting in suspension of translation initiation. As temperature increases, melting of the secondary structure causes the release of an SD sequence resulting in the resumption of translation [67].

A novel functionality was created based on a temperature-controlled element of Salmonella fused to a hammer-head ribozyme (HHR) that fractions itself to a liberated RBS and a thermo-sensitive hairpin connected to the HHR. It brought a temperaturecontrolled ribozyme forward - a so-called thermozyme. Therefore, at higher temperature, the self-cleavage activity of the thermometer structure is impaired and the gene expression is silenced. This is the opposite of the natural behavior of thermosensors [68].

Another simple theoretical thermometric mechanism was implemented for de novo design of a synthetic thermosensor based on an RBS embedded in a single stem loop that unmasks when the temperature reaches a predicted range. This hypothesis was assessed by constructing a particular modular structure including a promoter, a start codon, an SD sequence and a complementary anti-SD sequence (separated by four unique restriction sites), and standard reporter genes.

Another example is that the synthetic thermometer is designed based on the 5' UTR of the $c I I I$ gene of coli-phage $\lambda$, which switches between two secondary conformations (on/off structures) in response to temperature rather than fully melting. In the off conformation at low temperature, the RBS is veiled, but in the on conformation at higher temperatures, the RBS is revealed. Designed RNA thermometers subjected to extra mutations in practice lead to functional thermosensors that were actually temperature inducible using the reporter gene of lacZ (encoding $\beta$-galactosidase) $[66,69,70]$.

\section{Challenges and difficulties in using antisense RNA}

Although RNA regulatory systems have many applications, some obstacles remain.

\section{Method-born limitations in the procedure of small RNA identification}

Currently, classical procedures of small RNA identification rely on bioinformatical approaches, which are somehow limited to a predefined data pool. This may direct research to an incomplete library that displays bias to sequence conservation, specific promoters and Rho-independent terminators, while missing several other aspects, including ORFs, Rho-dependent terminators and Hfq-independent small RNAs. To ensure a global unbiased approach to small RNA identification, other experimental and predictive procedures such as transcriptional profiling with the whole genome, massively parallel sequencing, and targeted identification of small RNAs are suggested [71].

\section{Sufficient supplement of synthetic RNA for silencing}

Another difficulty of the practical application of RNA silencers, either naturally existing in the cell or delivered into the cell, is the sufficiency of the silencer agent for efficient 
silencing activity. Considering the abundance of mRNAs in the cell, an acceptable level of expression or delivery and stabilizing structures should be provided in order to achieve practical silencing [60].

Most delivery methods are culture dependent. Most prokaryotes from natural environments are not readily culturable. Moreover, even in culturable in vitro genetic manipulation methods, the nucleobase polymer transferring process is not $100 \%$ efficient. On the other hand, DNA and RNA transferred to natural microbial communities can change their functionality. However, in addition to their beneficial applications, the inevitable subsequences should also be considered [72].

\section{Delivery methods}

In the world of molecular biology, the success of every technique such as gene therapy and RNA regulation is highly dependent on the efficiency of the delivery approach of the multinucleotide molecule to the cell. A simple and efficient delivery system is necessary to exploit a prosperous RNA regulatory technology. Different biological physical and/or chemical methods are exerted for nucleotide polymers, as summarized in Table 4 [73-76].

Table 4 Delivery methods for transferring synthetic regulatory RNAs to bacteria

\begin{tabular}{|c|c|}
\hline Delivery methods & Traits \\
\hline \multicolumn{2}{|l|}{ Biological methods } \\
\hline Transduction & A specific DNA donor is required for DNA transfer to recipient bacteria \\
\hline \multirow[t]{2}{*}{ Conjugation } & $\begin{array}{l}\text { Requiring physical contact of recipient and donor (host strain) with a } \\
\text { conjugative plasmid or participation of a third bacterium with a helper plasmid }\end{array}$ \\
\hline & Not useful for large-scale delivery applications \\
\hline Gene transformation & Limited to a few naturally competent groups \\
\hline \multicolumn{2}{|l|}{ Physical methods } \\
\hline \multirow[t]{2}{*}{ Electroporation } & Highly efficient but requires low ionic strength medium and high voltage \\
\hline & Not useful for large-scale delivery applications \\
\hline Laser irradiation & $\begin{array}{l}\text { Employs a laser to change cell permeability to allow transferal; requires physical } \\
\text { contact of laser and cells }\end{array}$ \\
\hline $\begin{array}{l}\text { Ultrasound DNA delivery } \\
\text { (UDD); Sonoporation }\end{array}$ & $\begin{array}{l}\text { Appropriate approach for plasmid or DNA fragment transferal to eukaryotic cells } \\
\text { (e.g., gene therapy) }\end{array}$ \\
\hline Heat shock transfer & Mostly used for E. coli (in parallel with the calcium phosphate method) \\
\hline \multicolumn{2}{|l|}{ Chemical methods } \\
\hline \multirow[t]{2}{*}{ Protein \& Peptides } & $\begin{array}{l}\text { Introduced in the late } 1950 \text { s, this technique originally used high salt concentration and } \\
\text { polycationic proteins to enhance nucleic acid entry into the cell. }\end{array}$ \\
\hline & $\begin{array}{l}\text { Now cationic peptides are using to enhance nucleic acid delivery. Cationic } \\
\text { peptides have been found useful for enhancing cellular uptake and/or cell } \\
\text { targeting oligonucleotide analogs. These peptides are synthetically } \\
\text { conjugated, used as non-covalent complexes, or used in combination with } \\
\text { polymer formulation techniques }\end{array}$ \\
\hline Calcium phosphate & Simple, effective and still widely used for nucleic acid delivery \\
\hline Artificial lipids & $\begin{array}{l}\text { DNA has been successfully complexed with cationic, anionic and neutral } \\
\text { liposomes. These complexes can be handled easily, but lipid-based systems } \\
\text { generally have significant drawbacks, including the lack of targeting and } \\
\text { variations arising during fabrication }\end{array}$ \\
\hline Naonparticles & $\begin{array}{l}\text { Using carbon nanotubes, nucleic acid is delivered into cells. Magneto- } \\
\text { transformation has also been used for nucleic acid transfer, but in that } \\
\text { method, pulsed magnetic fields assisted the delivery of DNA using magnetic } \\
\text { nanoparticles. }\end{array}$ \\
\hline
\end{tabular}


The most commonly used methods include conjugation, electroporation and heat shock transfer (especially for E. coli). Other physical techniques are usually only employed for small-scale prokaryotic cells [77]. Ultrasound DNA delivery (UDD) is another practical method that can theoretically deliver DNA or RNA to any cell type (including bacterial, fungal, plant or mammalian cells) without the need for ion-free media and are thus usable for growing cells in natural media or human body fluids. Finally, it provides a non-invasive method allowing no necessity of direct physical contact [78]. However, the frequency ranges that are efficient for eukaryotic cells are less effective in prokaryotic cells compared to classic bacterial transformation methods [73].

\section{Conclusion}

The antisense RNA regulatory system is a mechanism for sequence-specific recognition of a particular transcript. It is usually involved in the degradation of said transcript. However, by definition, it could also be involved other forms of reduction or alteration of expression. New asRNA gene-controlling cases are being identified in nature, accompanied by the discovery of more mechanisms with a variety of potential applications. These utilities derive from variable mechanisms through which RNA regulatory systems influence target mRNA. AsRNAs can cause target mRNA degradation, change mRNA processing and/or affect mRNA transcription. The same applies to artificial asRNAs.

It remains to be seen whether transcriptional interference or antisense regulation can regulate sense-antisense pairs, but synthetic biology studies indicate that antisense transcription can improve existing networks by adding some constructive complexities. It is also possible to create new networks with desirable properties.

Regulatory RNA systems have considerable potential and are already used in silencing bacterial genes, altering bacterial behavior and inhibiting bacterial biofilm formation. They are also used as novel antibiotics, especially against multidrug-resistant bacteria. These applications have made them a popular subject for research.

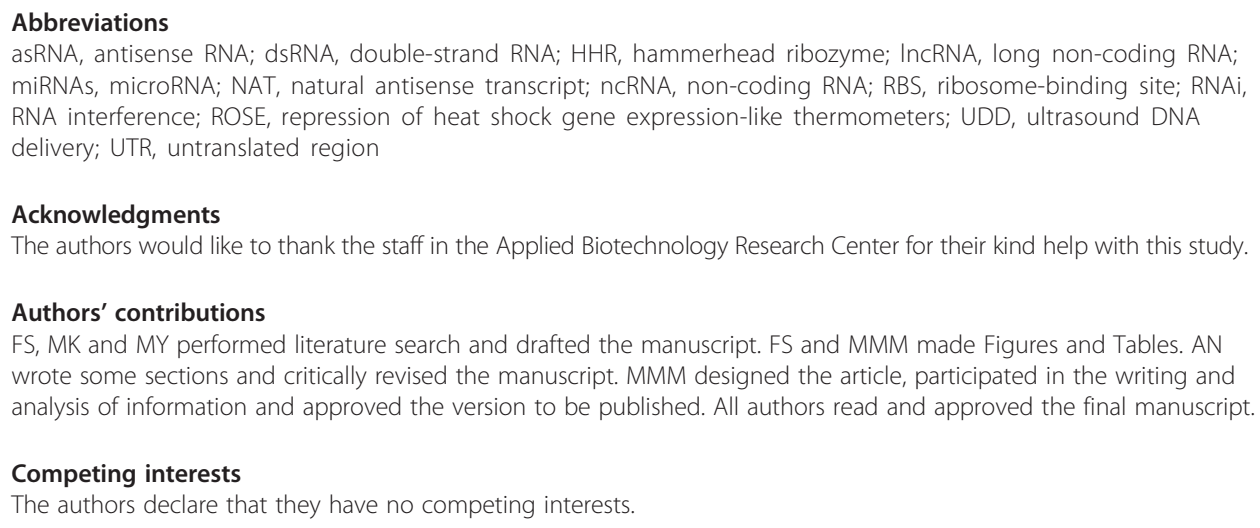


2. Wagner E, Flardh K. Antisense RNAs everywhere? Trends Genet. 2002;18:223-6.

3. Khorkova O, Myers A, Hsiao J, Wahlestedt C. Natural antisense transcripts. Hum Mol Gen. 2014;23:54-63.

4. Hindley J. Fractionation of 32P-labelled ribonucleic acids on polyacrylamide gels and their characterization by fingerprinting. J Mol Biol. 1967;30:125-36.

5. Vanhée-Brossollet C, Vaquero C. Do natural antisense transcripts make sense in eukaryotes? Gene. 1998;211:1-9.

6. Lybecker M, Bilusic I, Raghavan R. Pervasive transcription: detecting functional RNAs in bacteria. Transcription. 2014;5:e944039.

7. Numata K, Okada Y, Saito R, Kiyosawa H, Kanai A, Tomita M. Comparative analysis of cis-encoded antisense RNAs in eukaryotes. Gene. 2007;392:134-41.

8. Goh S, Stach J, Good L. Antisense effects of PNAs in bacteria. Methods Mol Biol. 2014;1050:223-36.

9. Cho KH, Kim JH. Cis-encoded non-coding antisense RNAs in streptococci and other low GC Gram (+) bacterial pathogens. Front Genet. 2015;6:110-5.

10. Rasmussen LCV, Sperling-Petersen HU, Mortensen KK. Hitting bacteria at the heart of the central dogma: sequence-specific inhibition. Microb Cell Fact. 2007;6:24-8.

11. Lapidot M, Pilpel Y. Genome-wide natural antisense transcription: coupling its regulation to its different regulatory mechanisms. EMB Rep. 2006;7:1216-22.

12. Isaacs FJ, Dwyer DJ, Collins JJ. RNA synthetic biology. Nat Biotechnol. 2006:24:545-54.

13. Caldelari I, Chao Y, Romby P, Vogel J. RNA-mediated regulation in pathogenic bacteria. Cold Spring Harbor perspect in medicine 2013;19:123-9

14. Thomason MK, Bischler T, Eisenbart SK, Forstner KU, Zhang A, Herbig A, et al. Global transcriptional start site mapping using differential RNA sequencing reveals novel antisense RNAs in Escherichia coli. J Bacteriol. 2015;197:18-28.

15. Duygu B, Poels EM, Da Costa Martins PA. Genetics and epigenetics of arrhythmia and heart failure. Front Genet. 2013;30:202-4.

16. Wagner EG. Kill the messenger: bacterial antisense RNA promotes mRNA decay. Nat Struct Mol Biol. 2009;16:804-6.

17. Georg J, Hess WR. cis-antisense RNA, another level of gene regulation in bacteria. Microbiol Mol Biol Rev. 2011;75:286-300

18. Stazic D, Lindell D, Steglich C. Antisense RNA protects mRNA from RNase E degradation by RNA-RNA duplex formation during phage infection. Nuc Acids Res. 2011;39:4890-9.

19. Green PJ, Pines O, Inouye M. The role of antisense RNA in gene regulation. Annu Rev Biochem. 1986;55:569-97.

20. Guillier M, Gottesman S, Storz G. Modulating the outer membrane with small RNAs. Genes Dev. 2006;20:2338-48.

21. Delihas N, Forst S. MicF: an antisense RNA gene involved in response of Escherichia coli to global stress factors. J Mol Biol. 2001;313:1-12.

22. Faghihi MA, Wahlestedt C. Regulatory roles of natural antisense transcripts. Nat Rev Mol Cell Biol. 2009;10:637-43.

23. Waters LS, Storz G. Regulatory RNAs in bacteria. Cell. 2009;136:615-28.

24. Kopf M, Klähn S, Scholz I, Hess WR, Voß B. Variations in the non-coding transcriptome as a driver of inter-strain divergence and physiological adaptation in bacteria. Sci Rep. 2015;5:9560-7.

25. Mok WW, McManus SA, Li Y. Small Size, Big Impact: Bacterial Functional Nucleic Acids and Their Applications. Chemical Biology of Nucleic Acids: Springer. 2014;40:309-323.

26. Sharma CM, Vogel J. Differential RNA-seq: the approach behind and the biological insight gained. Curr Opin Microbiol. 2014;19:97-105.

27. Thomason MK, Storz G. Bacterial antisense RNAs: how many are there and what are they doing? Annu Rev Genet 2010;44:167-88.

28. Liu SR, Hu CG, Zhang JZ. Regulatory effects of cotranscriptional RNA structure formation and transitions. Wiley interdiscip rev RNA. 2016;47:503-27.

29. Lasa I, Toledo-Arana A, Dobin A, Villanueva M, De Los Mozos IR, Vergara-Irigaray M, et al. Genome-wide antisense transcription drives mRNA processing in bacteria. Proc Natl Acad Sci U S A. 2011;108:20172-7.

30. Yang Z, Jin X, Rao X, Hu FA. Natural antisense transcript regulates mucD gene expression and biofilm biosynthesis in Pseudomonas aeruginosa. Microbiol. 2011:80:768-74.

31. Wagner EGH, Simons RW. Antisense RNA control in bacteria, phages, and plasmids. Annu Rev Microbiol. 1994;48:713-42.

32. Chen S, Zhang A, Blyn LB, Storz G. MicC, a second small-RNA regulator of Omp protein expression in Escherichia coli. J Bacteriol. 2004;186:6689-97.

33. Sillers R, Al-Hinai MA, Papoutsakis ET. Aldehyde-alcohol dehydrogenase and/or thiolase overexpression coupled with COA transferase downregulation lead to higher alcohol titers and selectivity in Clostridium acetobutylicum fermentations. Biotechnol Bioeng. 2009;102:38-49.

34. Wagner EGH, Altuvia S, Romby P. 12 Antisense RNAs in bacteria and their genetic elements. Adv Genet. 2002:46:361-98.

35. Faridani OR, Nikravesh A, Pandey DP, Gerdes K, Good L. Competitive inhibition of natural antisense Sok-RNA interactions activates Hok-mediated cell killing in Escherichia coli. Nucleic Acids Res. 2006;34:5915-22.

36. Kawano M, Aravind L, Storz G. An antisense RNA controls synthesis of an SOS-induced toxin evolved from an antitoxin. Mol Microbiol. 2007;64:738-54.

37. Kawano M. Divergently overlapping cis-encoded antisense RNA regulating toxin-antitoxin systems from E. coli: hok/sok, Idr/rdl, symE/symR. RNA Biol. 2012;9:1520-7.

38. Moghaddam MM, Khodi S, Mirhosseini A. Quorum Sensing in Bacteria and a Glance on Pseudomonas aeruginosa. Clin Microbial. 2014;3:1-10.

39. Aghamollaei H, Moghaddam MM, Kooshki H, Heiat M, Mirnejad R, Barzi NS. Detection of Pseudomonas aeruginosa by a triplex polymerase chain reaction assay based on lasl/R and gyrB genes. J Infec Public Health. 2015;8:314-22.

40. Romby P, Vandenesch F, Wagner EGH. The role of RNAs in the regulation of virulence-gene expression. Curr Opin Microbiol. 2006;9:229-36.

41. Liu JM, Camilli A. A broadening world of bacterial small RNAs. Curr Opin Microbiol. 2010;13:18-23.

42. Courtney C, Chatterjee A. cis-Antisense RNA and Transcriptional Interference: Coupled Layers of Gene Regulation. J Gene Ther. 2014;2:9-15. 
43. Papenfort K, Vogel J. Regulatory RNA in bacterial pathogens. Cell Host Microbe. 2010;8:116-27.

44. Delihas N. Regulation of gene expression by trans-encoded antisense RNAs. Mol Microbiol. 1995;15:411-4.

45. Gottesman S. The small RNA regulators of Escherichia coli: roles and mechanisms. Annu Rev Microbiol. 2004;58:303-28

46. Qin D, Xu C. Study strategies for long non-coding RNAs and their roles in regulating gene expression. Cell Mol Biol Letters. 2015:20:323-49.

47. Wahlestedt C. Natural antisense and noncoding RNA transcripts as potential drug targets. Drug Discov Today. 2006;11:503-8.

48. Wall ME, Hlavacek WS, Savageau MA. Design of gene circuits: lessons from bacteria. Nat Rev Genet. 2004;5:34-42.

49. Kemmer C, Neubauer P. Antisense RNA based down-regulation of RNaseE in E. coli. Microb Cell Fact. 2006;5:38-44.

50. Tummala SB, Junne SG, Papoutsakis ET. Antisense RNA Downregulation of Coenzyme A Transferase Combined with Alcohol-Aldehyde Dehydrogenase Overexpression Leads to Predominantly Alcohologenic Clostridium acetobutylicum Fermentations. J Bacteriol. 2003;185:3644-53.

51. Desai RP, Papoutsakis ET. Antisense RNA strategies for metabolic engineering of Clostridium acetobutylicum. Appl Environ Microbiol. 1999;65:936-45.

52. Bouazzaoui K, LaPointe G. Use of antisense RNA to modulate glycosyltransferase gene expression and exopolysaccharide molecular mass in Lactobacillus rhamnosus. J Microbiol Methods. 2006;65:216-25.

53. Sturino JM, Klaenhammer TR. Engineered bacteriophage-defence systems in bioprocessing. Nat Rev Microbiol. 2006:4:395-404.

54. Ghafourian S, Raftari M, Sadeghifard N, Sekawi Z. Toxin-antitoxin systems: classification, biological function and application in biotechnology. Curr Issues Mol Biol. 2014;6:9-14.

55. Alessandra S, Flavio S. BMB reports: Artificial antisense RNAs silence LacZ in E. coli by decreasing target mRNA concentration. Biochem Mol Biol Rep. 2008;41:568-84.

56. Singh SB, Phillips JW, Wang J. Highly sensitive target-based whole-cell antibacterial discovery strategy by antisense RNA silencing. Curr Opin Drug Discov Dev. 2007;10:160-5.

57. Genilloud $\mathrm{O}$. The re-emerging role of microbial natural products in antibiotic discovery. Antonie Van Leeuwenhoek. 2014;106:173-88.

58. Nikravesh A, Dryselius R, Faridani OR, Goh S, Sadeghizadeh M, Behmanesh M, et al. Antisense PNA accumulates in Escherichia coli and mediates a long post-antibiotic effect. Mol Ther. 2007;15:1537-42.

59. Ji Y, Yin D, Fox B, Holmes DJ, Payne D, Rosenberg M. Validation of antibacterial mechanism of action using regulated antisense RNA expression in Staphylococcus aureus. FEMS Microbiol Lett. 2004;231:177-84.

60. Good L, Stach JE. Synthetic RNA silencing in bacteria - antimicrobial discovery and resistance breaking. Front Microbiol. 2011;2:185-94

61. Liang JC, Bloom RJ, Smolke CD. Engineering biological systems with synthetic RNA molecules. Mol Cell. 2011:43:915-26.

62. Hong P, Li W, Li J. Applications of aptasensors in clinical diagnostics. Sensors. 2012;12:1181-93.

63. Said N, Rieder R, Hurwitz R, Deckert J, Urlaub H, Vogel J. In vivo expression and purification of aptamer-tagged small RNA regulators. Nucleic Acids Res. 2009;37:e133.

64. Wu Z, Liu X, Liu L, Deng H, Zhang J, Xu Q, et al. Regulation of IncRNA expression. Cell Mol Biol Lett. 2014;19:561-75.

65. Qi L, Lucks JB, Liu CC, Mutalik VK, Arkin AP. Engineering naturally occurring trans-acting non-coding RNAs to sense molecular signals. Nucleic Acids Res. 2012;40:5775-86.

66. Neupert J, Karcher D, Bock R. Design of simple synthetic RNA thermometers for temperature-controlled gene expression in Escherichia coli. Nucleic Acids Res. 2008;36:e124.

67. Lavelle C, Busi F, Arluison V. Multiple Approaches for the Investigation of Bacterial Small Regulatory RNAs Selfassembly. RNA Nanotechnology and Therapeutics: Springer. 2015;10:21-42.

68. Saragliadis A, Krajewski SS, Rehm C, Narberhaus F, Hartig JS. Thermozymes: Synthetic RNA thermometers based on ribozyme activity. RNA Biol. 2013;10:1009-16.

69. Neupert J, Bock R. Designing and using synthetic RNA thermometers for temperature-controlled gene expression in bacteria. Nat Protoc. 2009;49:1262-73.

70. Waldminghaus T, Kortmann J, Gesing S, Narberhaus F. Generation of synthetic RNA-based thermosensors. Biol Chem. 2008;389:1319-26.

71. Hebert CG, Valdes JJ, Bentley WE. Beyond silencing-engineering applications of RNA interference and antisense technology for altering cellular phenotype. Curr Opin Biotechnol. 2008;19:500-5.

72. Chang TH, Wu LC, Lin JH, Huang HD, Liu BJ, Cheng KF, et al. Prediction of small non-coding RNA in bacterial genomes using support vector machines. Expert Syst Appl. 2010;37:5549-57.

73. Song Y, Hahn T, Thompson IP, Mason TJ, Preston GM, Li G, et al. Ultrasound-mediated DNA transfer for bacteria. Nucleic Acids Res. 2007:35:e129.

74. Luo D, Saltzman WM. Synthetic DNA delivery systems. Nat Biotechnol. 2000;18:33-7.

75. Järver P, Coursindel T, Andaloussi SE, Godfrey C, Wood MJ, Gait MJ. Peptide-mediated cell and in vivo delivery of antisense oligonucleotides and siRNA. Mol Ther Nucleic Acids. 2012;1:e27.

76. Saei AA, Barzegari A, Majd MH, Asgari D, Omidi Y. Fe3O4 nanoparticles engineered for plasmid DNA delivery to Escherichia coli. J Nanopart Res. 2014;16:1-11.

77. Heiat M, Aghamollaei H, Moghaddam MM, Kooshki H. Using CM11 peptide as a cell Permeable agent for the Improvement of conventional plasmid transformation methods in Escherichia coli and Bacillus subtilis. Minerva Biotecnol. 2014;26:149-57.

78. Shepherd J, Ibba M. Bacterial transfer RNAs. FEMS Microbiol Rev. 2015;39:280-300. 